\title{
Image Denoising using a Novel Frobenius Norm Filter for a Class of Noises
}

\author{
Sutanshu Saksena Raj \\ USIT, GGS IP University \\ Delhi, India
}

\author{
Palak Jain \\ IGIT, GGS IP University \\ Delhi, India
}

\author{
Divij Babbar \\ ESIEE \\ Paris, France
}

\begin{abstract}
In this paper, we present a novel Frobenius Norm Filter (FNF), which is a spatially selective noise filtration technique in the wavelet subband domain. We address the issue of denoising of images corrupted with additive, multiplicative, and uncorrelated noise. The proposed nonlinear filter is an adaptive order statistic filter functioning on the $L^{2}$ space, which modulates itself according to the noise level. We have applied the comparative Frobenius Norm under a given window set and pixel connectivity for removal of noise. We prove the existence of a minimizer, and its convergence, of our specialized filter. We present a comparative study between FNF and certain standard filters, and find that our method is capable of reducing large noise blotches, and is an adequate preprocess to improving the quality of segmentation and facilitating the feature extraction process. We obtained better restoration results, especially when the images were highly corrupted and having a high noise density.
\end{abstract}

Keywords: Frobenius Norm Filter, Adaptive Order Statistics, Class of Noises, Wavelet Subband Domain.

\section{INTRODUCTION}

Wavelet transforms have been utilized effectively for image denoising, providing a means to exploit the relationships between coefficients at multiple scales [15]. A key feature of such denoising techniques is the ability to accurately estimate the local variance of the wavelet coefficients and the associated noise component. Denoising of images corrupted by a class of un-correlated noise using wavelet techniques is very effective because of its ability to capture the energy of a signal in few energy transform values [12].

Our work was primarily motivated by the desire to devise a filtration technique based on Relational Context Spatial Domain Analysis for the removal of additive, uncorrelated and multiplicative noise. We present a comprehensive study of the filtering abilities of the novel Frobenius Norm Filter when applied to a class of noises, and compare its performance with that of the Adaptive Median Filter and the conventional Median Filter. We were especially interested in Biorthogonal Filters because they constitute an important subclass of regular, perfect reconstruction FIR filters that permit the analysis and synthesis filters to be linear phase [7].

Noise models can be considered as random variables characterised by their Probability Density Functions (PDF). The proposed filter modulates itself according to the PDF associated with each subband of the transformed noisy data. We have used the following Noise PDF's in our paper - Laplace noise, Rayleigh noise, Erlang noise, Poisson noise and Speckle noise. Poisson noise is not additive, but is image pixel-intensity dependent [9], therefore, it is not easy to establish an appropriate statistical model for the noise by simply examining the corrupted image. Similar is the case of Speckle noise, which is a 'multiplicative' noise [17]. The Rayleigh Density is useful in characterizing noise phenomena in range imaging and the Erlang Noise model finds its application in laser imaging [2].

The data representing the image $S: \Omega \rightarrow \mathbb{R}_{+}, \Omega \subset \mathbb{R}^{2}$ is corrupted individually from a class of noises. Such a noise severely degrades the image. In order to restore the image, several independent measurements for the same scene should be realized, thus yielding a set of data

$$
\sum_{k}=S \eta_{k}+n_{k}, \forall k \in\{1, \ldots K\}, K \geq 1,
$$

where $\eta_{k}: \Omega \rightarrow \mathbb{R}_{+}$and $n_{k}$ represent the additive, multiplicative and un-correlated noise relevant to each measurement $k$ of the wavelet decomposition structure. Minimization of outlier effects can be accomplished by replacing the above mentioned linear form (1), with $\hat{S}(x, y)=$ Frobenius Norm $\left\{\sum_{k}\right\}$, such that the PSNR value is maximum; subject to optical evaluation [14]. Histogram Matching is also accounted for. The Frobenius Norm comes from an inner product on the space of all matrices in $K$, where $K$ denotes the field of real or complex numbers. The Median defined via this Norm is:

$$
\operatorname{med}_{F}\left(A_{1, \ldots,} A_{n}\right)=\min _{X \in \mathcal{L}^{d}} \sum_{i=1}^{n}\left\|X-A_{i}\right\|_{F} .
$$

with vectors in $\mathbb{R}^{d^{2}}$ and symmetric matrices in $\mathcal{L}^{d}$, which is the convex optimization cone of vectors $\left\{x \in \mathbb{R}^{d} \mid x_{d} \geq\right.$ $\left.\sqrt{\left(x_{1}\right)^{2}+\cdots+\left(x_{d-1}\right)^{2}}\right\}$, while $\mathcal{S}^{d}$ is the linear space of symmetric $d \times d$ real matrices [13]. Arbitration between the perpetuation of diagnostic information and noise suppression must be balanced in images, so as to reduce errors which will propagate in the neighborhood regions.

\section{MATHEMATICAL BACKGROUND}

The Frobenius Norm can be computed from the matrix entries $a_{j, k}, j, k=1, \ldots d$ of matrix $A$ having Eigen Values $\lambda_{1}(A), \ldots \lambda_{d}(A)$ as:

$$
\|A\|_{F}=\sqrt{\sum_{j=1}^{d}\left|\lambda_{j}(A)\right|^{2}}=\sqrt{\sum_{j, k}^{d} a_{j, k}^{2}}
$$


Let $\Omega \subset \mathbb{R}^{2}$ denote an open bounded set of $\mathbb{R}^{2}$ with Lipschitz boundary. $B V(\Omega)$ is the subspace of functions $S \in L^{1}(\Omega)$ such that the following quantity is finite:

$$
\begin{gathered}
J(S)=\sup \left\{\int_{\Omega} S(x) \operatorname{div}(\xi(x)) d x / \xi \in C_{0}^{\infty}\left(\Omega, \mathbb{R}^{2}\right),\right. \\
\left.\|\xi\|_{L^{\infty}\left(\Omega, \mathbb{R}^{N}\right)} \leq 1\right\}
\end{gathered}
$$

where $\xi \in \Omega, \psi: \mathbb{R}_{+} \rightarrow \mathbb{R}_{+}$assesses closeness to data. $B V(\Omega)$ endowed with norm $\|S\|_{B V}=\|S\|_{L^{1}}+J(u)$ is a Banach Space [16]. If $S \in B V(\Omega)$, the distributional derivative $D S$ is a bounded Radon Measure. Also, if $1 \leq p \leq 2$, we have $B V(\Omega) \subset L^{p}(\Omega)$ and the given embedding is compact.

Since $B V(\Omega) \subset L^{2}(\Omega)$, we can extend the functional $J$ over $L^{2}(\Omega)$, hence we have:

$$
J(S)=\left\{\begin{array}{c}
\int_{\Omega}|D S| \text { if } S \in B V(\Omega) \\
\infty \text { if } S \in L^{2}(\Omega) \backslash B V(\Omega)
\end{array}\right.
$$

We can then define the sub-differential $\partial J$ of $J$ as $n_{k} \in$ $\partial J(u)$ iff for all $w \in L^{2}(\Omega)$, we have $J(S+w) \geq J(S)+$ $\langle S, w\rangle_{L^{2}(\Omega)}$ where $\langle\cdot\rangle$ denotes the inner product. What is important to us is that one can determine whether a function is in $B V\left(L_{p}(\Omega)\right)$ simply by examining its wavelet coefficients [13]. For $S_{i, j}$ at the boundary, Neumann's condition is used.

Considering the convex optimisation problem and introducing $n$ additional variables $t=\left(t_{1} \ldots t_{n}\right)^{T}$, we rewrite the Frobenius Median (defined earlier) as:

$$
\inf _{X \in \mathcal{S}^{d}, t \in \mathbb{R}^{n}}\langle e, t\rangle, \quad\left\|X-A_{i}\right\|_{2} \leq t_{i}, i=1 \ldots n
$$

where $e$ denotes the vector $(1 \ldots 1)^{T} \in \mathbb{R}^{n}$ and each constraint is convex because $\left(X^{T}, t_{i}\right)^{T}$ varies in the convex cone $\mathcal{L}_{i}^{d^{2}+1}$ [2]. If the corresponding convex constraint sets are $\mathcal{G}_{i}, i=1 \ldots n$, Frobenius Median problem becomes:

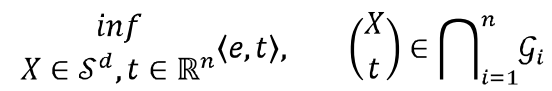

This optimization problem is convex, since the objective function is linear, and since the intersection of convex sets is convex, too. Since a matrix, $\mathrm{X}$, outside the convex assembly is not the minimiser of the Norm, therefore, the Frobenius Median of a positive semi-definite symmetric matrix is also positive semi-definite. Since the Frobenius Norm works on the $L^{2}$ space, as do the Wavelets [4]; we combine both to propose a De-noising algorithm.

\section{DISCUSSION \& DESCRIPTION}

The Frobenius Norm works on Eigen values, Eigen vectors, and the Matrix Space, which are unique to a given system. When applied to a neighbourhood of pixels, the connectivity is preserved even when the image is highly corrupted. This gives good results even when the window size is large. The Frobenius Norm is a sub-multiplicative measure, i.e., $\|A B\|_{F} \leq\|A\|_{F}\|B\|_{F}$, and is useful for adaptive optimization of a denoising algorithm where the noise model's PDF can be measured within the $L^{2}(\Omega)$ Norm. If the PDF satisfies the Frobenius $L^{2}(\Omega)$ norm, i.e., the calculated values lie in the closed disc corresponding to the Frobenius Theorem, then the FNF replaces the corrupted pixel with the value returned by Frobenius Norm. This comparative approach lends flexibility to the Frobenius Norm Filter and it is successful in tackling different class of noises. Most set of noises are compatible with the FNF as they are measured either in the $L^{1}(\Omega)$ or $L^{2}(\Omega)$ space. For those that are not, the FNF algorithm assumes that the image edges are joined to form a virtual surface. Our algorithm essentially involves operations between neighbouring pixels. Propriety methods such as 4connectivity and 8-connectivity are not used as they lead to undesirable topological anomalies [10]. It uses connectivity in the spatial domain and exploits the group behaviour of the pixel neighbourhood. This Norm is often easier to compute than Induced Norms [7].

A Matrix acts on certain Vectors, called Eigenvectors, by changing only their magnitude, and leaving their direction unchanged. It does so by multiplying the magnitude of the Eigenvector with a factor, which is either positive or negative, called Eigenvalue. The Eigen values of some matrices are sensitive to perturbations. Every $L^{2}$ space consists of equivalence classes of functions and we can think of an $L^{2}$ function as a density function. A Noise Model's PDF, measured either in the $L^{1}(\Omega)$ or $L^{2}(\Omega)$ space, is compatible with the Frobenius $L^{2}\left(\mathbf{R}^{n}\right)$ Norm.

The central pixel's output value is dependent on the local statistical information [9]. Frobenius Norm filter adapts itself to the local properties, information surrounding a central pixel in order to calculate a new pixel value. Frobenius Norm filter is much better in preservation of the image sharpness, details and edges (sharp contrast variation), while suppressing Noise [16].

\subsection{Proposed Algorithm}

Let $x_{\mathrm{i}, \mathrm{j}}$, for $(i, j) \in A \equiv\{1, \ldots, M\} \times\{1, \ldots, N\}$, be the gray level of a true M-by-N image $x$ at the pixel location $(i, j)$, and $\left[s_{\min }, s_{\max }\right]$ be the dynamic range of $x$, i.e $s_{\min } \leq$ $x_{\mathrm{i}, \mathrm{j}} \leq s_{\max }$ for all $(i, j) \epsilon A$. Denote by $y$ a noisy image. Let $C_{i, j}^{w}$ be a window of size $w \times w$ centered at $(i, j)$, i.e

$$
C_{i, j}^{w}=\{(k, l):|k-i|<w \text { and }|j-i| \leq w\}
$$

and let $w_{\max } \times w_{\max }$ be the maximum window size. The algorithm tries to identify the noisy pixels $y_{i, j}$, and then replace each $y_{i, j}$ by the Frobenius Norm of the pixels in $C_{i, j}^{w}$.

Algorithm (FNF): For each pixel location $(i, j)$, do

1. Initialize $\mathrm{w}=3$.

2. Compute $s_{i, j}^{\min , w}, s_{i, j}^{\text {fro,w }}$, and $s_{i, j}^{\max , w}$, which are the minimum, Frobenius Norm and maximum of the pixel values in $C_{i, j}^{w}$, respectively.

3. Compute $s_{i, j}^{f r o, w}=\operatorname{sqrt}\left(\operatorname{sum}\left(\operatorname{diag}\left(w^{\prime} * w\right)\right)\right)$.

4. If $s_{i, j}^{\min , w}<s_{i, j}^{\text {fro,w }}<s_{i, j}^{\max , w}$ then go to step 5, otherwise set $\mathrm{i}=\mathrm{i}+1$. 
5. If $s_{i, j}^{\min , w}<y_{i, j}<s_{i, j}^{\max , w}$, then $y_{i, j}$ is not a noisy candidate, else we replace $y_{i, j}$ by $s_{i, j}^{\text {fro,w }}$.

We denote as $x(n, m)$ a neighborhood of coefficients clustered around this reference coefficient. In general, the neighborhood may include coefficients from other subbands (i.e., corresponding to basis functions at nearby scales and orientations), as well as from the same subband. In our case, we use a neighborhood of coefficients drawn from two subbands at adjacent scales, thus taking advantage of the strong statistical coupling observed through scale in multiscale representations which are innate to the Wavelet Transform [15].

\section{EXPERIMENTAL RESULTS}

The standard image ( $N=256^{2}$ pixels) is corrupted with a class of different noises ranging from additive, multiplicative and impulsive noise. The noisy images were then processed by the FNF with the parameter settings shown in Table 2. To assess the performance of Frobenius Norm Filtering method, it is compared with the Adaptive Median Filtering (AMF) method and the conventional Median filtering method. See Table 1. for the results, with $\mathrm{d}=0.05$ at various level of decompositions (n). To benchmark against the best possible performance of the filtering method, these parameters have been taken into consideration: the PSNR values and the visual quality of the image. Refer to Fig. 1 and 2 for illustrations. We also used a varying degree of noise density (d) in Fig. 3 to showcase the generic results.

The calculation of the square root of the summation of squared distance between the image's neighbouring pixels is the most computationally intensive procedure in the algorithm, but it is unique to an image and is lesser than AMF's temporal complexity [1] of $\mathcal{O}(N \log (N))$. The conventional median filter and its variants are essentially two-phase processes which are difficult to treat analytically [3].

One property of medians, which causes quite an inconvenience, is that it is independent of the norm, thereby increasing the computationally complexity [6]. Another observation is that the performance of the algorithm was consistent across the whole test data set. Wavelet Transform is applied to smoothen the de-noised image. The filter adopted was the 'bior6.8' filter and the ' $\mathrm{db} 2$ ' filter (see MATLAB), [11].
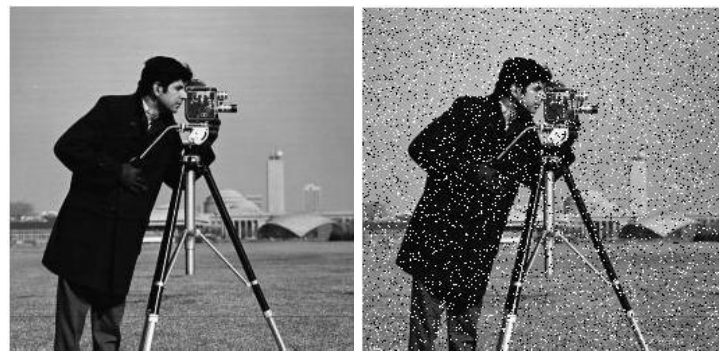
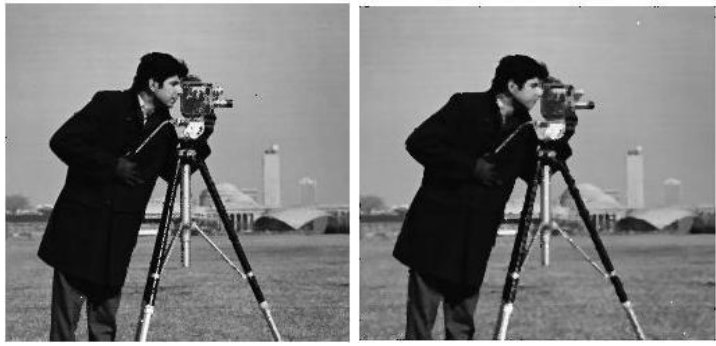

Figure 1. (Row-1: From L to R) (a) Original Image (b) Image corrupted with Impulse Noise, $d=0.1$.

(Row-2: From L to R) (c) Frobenius Norm Filtered (FNF) Image (d) Median Filtered Image.
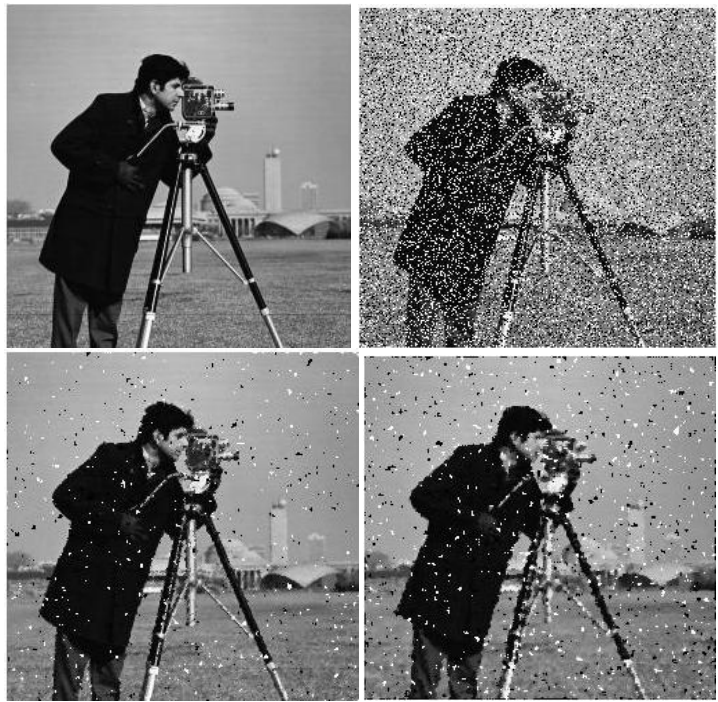

Figure 2. (Row-1: From L to R) (a) Original Image (b)

Image corrupted with Impulse Noise, $d=0.4$.

(Row-2: From L to R) (c) Frobenius Norm Filtered (FNF) Image (d) Median Filtered Image.

The experimental evaluation showed that the proposed Frobenius Norm Filtering method showed better results than the conventional Median Filtering \& Adaptive Median Filtering methods when the images were highly corrupted and having noise density $d \in[0.4,1)$, as can be seen from Figure 3. This Filter has been evaluated with several standard images, generating successful denoising results. Our results show that the novel Frobenius Norm Filter yields modest but consistent improvements over all competing methods.

Table 1. Comparative performance study of Denoising algorithms, in terms of PSNR (dB), for various filtering techniques, at $\mathrm{d}=0.05$.

\begin{tabular}{|c|c|c|}
\hline $\begin{array}{l}\text { Wavelet } \\
\text { Type }\end{array}$ & bior6.8 & $\mathrm{db} 2$ \\
\hline MEDIAN FILTERING & & \\
\hline $\mathrm{n}=2$ & 26.57 & 26.48 \\
\hline $\mathrm{n}=3$ & $\mathbf{2 6 . 8 1}$ & $\mathbf{2 6 . 7 9}$ \\
\hline
\end{tabular}




\begin{tabular}{|c|c|c|}
\hline $\mathrm{n}=4$ & 25.67 & 25.58 \\
\hline $\begin{array}{c}\text { ADAPTIVE MEDIAN } \\
\text { FILTERING (AMF) }\end{array}$ & & \\
\hline $\mathrm{n}=2$ & $\mathbf{2 9 . 7}$ & $\mathbf{2 9 . 9 2}$ \\
\hline $\mathrm{n}=3$ & 29.56 & 29.77 \\
\hline $\mathrm{n}=4$ & 28.32 & 28.11 \\
\hline $\begin{array}{c}\text { FROBENIUS NORM } \\
\text { FILTERING (FNF) }\end{array}$ & & \\
\hline $\mathrm{n}=2$ & 28.56 & 28.42 \\
\hline $\mathrm{n}=3$ & $\mathbf{2 8 . 6 4}$ & $\mathbf{2 8 . 6}$ \\
\hline $\mathrm{n}=4$ & 27.56 & 27.59 \\
\hline
\end{tabular}
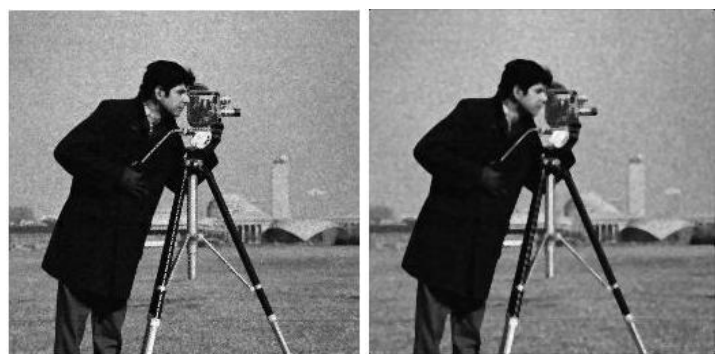

Figure 4. (Row-1: From L to R) (a) Original Image (b) Image corrupted with Poisson Noise. (Row-2: L to R) (c) Frobenius Norm Filtered (d) Median Filtered.

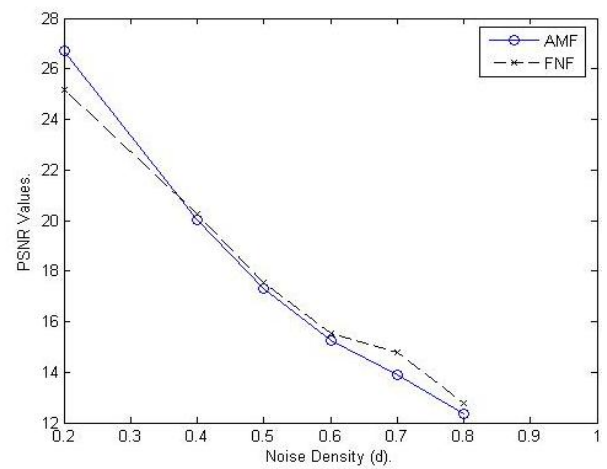

Figure 3. Graphical Plot of AMF and FNF at different values of $d$ for bior6.8

It was observed, without any loss of generality and independent of the noise model, that the FNF was exceptionally good at filtering objects / patches in the image whose respective dimensions were approximately 7 times or more than the dimension of the window size, $w$. Refer Fig. 4 and 5. Compatibility, in terms of de-noising, with noises having PDF's measurable in the $L^{2}(\Omega)$ norm is evident from the results. The filter performs well both with lower noise levels and higher noise levels.

Table 2. Parameter setting for the Simulations.

\begin{tabular}{|l|l|l|}
\hline \multicolumn{1}{|c|}{ Parameters } & Mean $(\mu)$ & Variance $\left(\sigma^{2}\right)$ \\
\hline Laplace Noise & & \\
\hline Erlang Noise & 0 & 0.01 \\
\hline Rayleigh Noise & 0.30 & 0.25 \\
\hline Speckle Noise & 0 & 0.0215 \\
\hline Salt \& Pepper & \multicolumn{2}{|c|}{$d$ (Noise Density) $=0.5$} \\
\hline
\end{tabular}

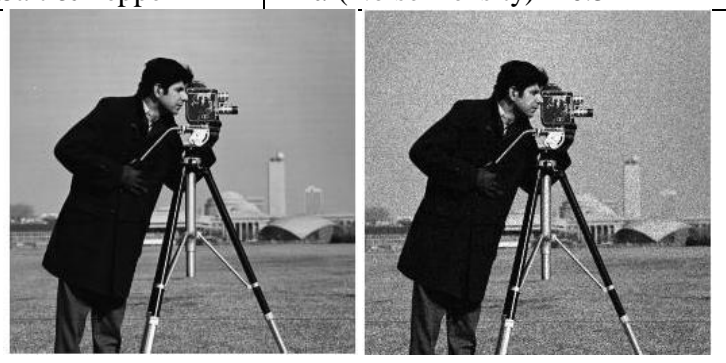

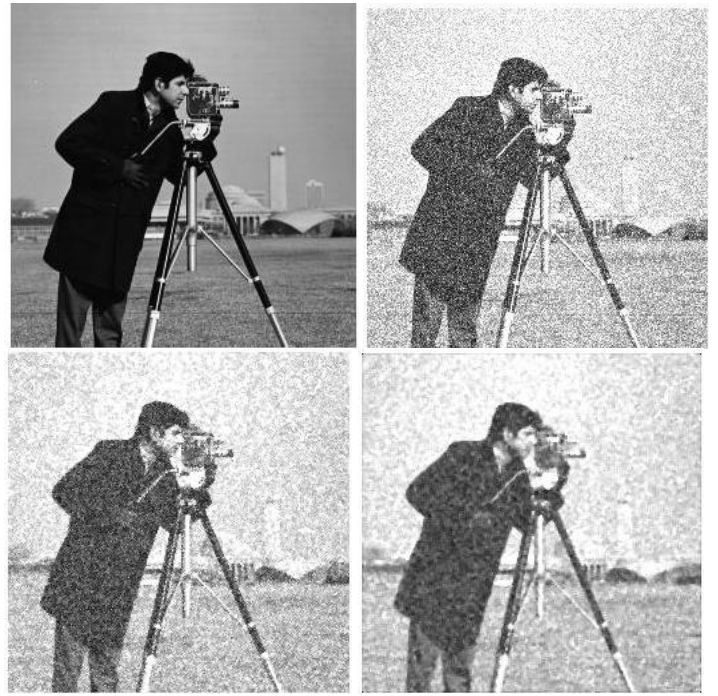

Figure 5. (Row-1: From L to R) (a) Original Image (b) Image corrupted with Rayleigh Noise. (Row-2: L to R) (c) Frobenius Norm Filtered (d) Median Filtered.

Overall our results confirm that the portrayal of salient image features such as edges and contours is consistently improved while no perceivable artifacts are introduced. Frobenius Norm is based on the analysis of the statistical properties of the noise model and does not involve calculations more than $\mathcal{O}(n)$ unlike the AMF. Thus it is computationally more efficient than Adaptive Median Filter [14]. It gives good results even when the window size is large. It is observed that they provide an adaptive edgepreserving regularity as well. There is no over-smoothing of edges or smudging within the periphery of the image. Refer Table 3 and 4 for results with a class of noises.

Table 3. Denoising Results (PSNR - dB) for various Noise Models at different decomposition levels.

\begin{tabular}{|c|c|c|c|}
\hline Filter & $\begin{array}{c}\text { PSNR } \\
\text { Values }(\mathrm{dB}) \\
\text { for } \mathrm{n}=2\end{array}$ & $\begin{array}{c}\text { PSNR } \\
\text { Values }(\mathrm{dB}) \\
\text { for } \mathrm{n}=3\end{array}$ & $\begin{array}{c}\text { PSNR } \\
\text { Values }(\mathrm{dB}) \\
\text { for } \mathrm{n}=4\end{array}$ \\
\hline \multicolumn{4}{|c|}{ Salt \& Pepper } \\
\hline Median & 17.29 & 16.99 & 17.14 \\
\hline ADP Med. & 17.45 & 17.21 & 17.34 \\
\hline Frobenius & $\mathbf{1 7 . 8 1}$ & 17.99 & 17.96 \\
\hline
\end{tabular}




\begin{tabular}{|l|c|c|c|}
\hline \multicolumn{4}{|c|}{ Erlang Noise } \\
\hline Median & 15.93 & 15.09 & 15.01 \\
\hline ADP Med. & 15.54 & 15.27 & 15.19 \\
\hline Frobenius & $\mathbf{1 6 . 6 1}$ & $\mathbf{1 6 . 4 4}$ & $\mathbf{1 6 . 3 1}$ \\
\hline \multicolumn{4}{|c|}{ Rayleigh Noise } \\
\hline Median & 15.49 & 15.47 & 15.46 \\
\hline ADP Med. & 15.59 & 15.56 & 15.54 \\
\hline Frobenius & $\mathbf{1 5 . 6 7}$ & $\mathbf{1 5 . 6 3}$ & $\mathbf{1 5 . 5 7}$ \\
\hline
\end{tabular}

\section{CONCLUSION}

A comparative performance evaluation has been presented between the novel Frobenius Norm Filter and its counterparts. A standard image is corrupted with different types of Noises, and the denoising effect of the filter is measured. The main elements of the algorithm were its spatial selectivity, adaptive order statistic nature and comparative approach. The FNF's reduction in Gibbs's ringing; rectification to alleviate blurring and the unnoticeable loss in spatial resolution render it a strong contender among the conventional denoising filters. The FNF is a refined approach that shows much promise as a pre-filter for reducing noise before performing image analysis and feature detection.

It is further suggested that the Frobenius Norm Filtering method be extended to a class of images like SAR, Medical, Laser, etc to check the denoising performance, as the obtained numerical results in this paper, both in objective and subjective terms, are really encouraging and the FNF outperform the most recent methods in this field. FNF's good Localization Characteristic and Protection of Sharp Edges will allow the Wavelet Filters to be very competitive in Edge Detection, Pattern Recognition, and Computer Vision.

Table 4. Denoising Results (PSNR - dB) for various Noise Models at different decomposition levels.

\begin{tabular}{|c|c|c|c|}
\hline $\mathrm{F}$ & $\begin{array}{c}\text { PSNR } \\
\text { Values }(\mathrm{dB}) \\
\text { for } \mathrm{n}=2\end{array}$ & $\begin{array}{c}\text { PSNR } \\
\text { Values }(\mathrm{dB}) \\
\text { for } \mathrm{n}=3\end{array}$ & $\begin{array}{c}\text { PSNR } \\
\text { Values }(\mathrm{dB}) \\
\text { for } \mathrm{n}=4\end{array}$ \\
\hline \multicolumn{4}{|c|}{ Laplace Noise } \\
\hline Median & 11.48 & 11.45 & 11.44 \\
\hline ADP Med. & 11.55 & 11.53 & 11.51 \\
\hline Frobenius & 11.59 & 11.56 & 11.54 \\
\hline \multicolumn{4}{|c|}{ Speckle Noise } \\
\hline Median & 23.38 & 23.71 & 22.99 \\
\hline ADP Med. & 23.15 & 22.65 & 22.28 \\
\hline Frobenius & 23.67 & 23.91 & 23.39 \\
\hline \multicolumn{4}{|c|}{ Poisson Noise } \\
\hline Median & 26.20 & 26.41 & 25.48 \\
\hline ADP Med. & 27.99 & 27.67 & 27.09 \\
\hline Frobenius & 28.26 & 28.43 & 27.51 \\
\hline
\end{tabular}

\section{REFRENCES}

[1] D. T. Kuan, et al, Adaptive Noise smoothing filter for Images with signal-dependent Noise, IEEE Trans. on Pattern Anal. And Machine Intell., vol. 7, pp. 165, 1985.
[2] E. Kolaczyk, Wavelet shrinkage estimation of certain Poisson intensity signals using corrected thresholds, Statist. Sinica, 9, pp. 119\{135, 1999.

[3] G. Demoment, "Image reconstruction and restoration: Overview of common estimation structures and problems," IEEE Trans. Acoustic, Speech, Signal Processing, vol. 37, no. 12, pp. 2024-2036, 1989.

[4] I. Daubechies, Ten Lectures on Wavelets, Vol. 61 of Proc. CBMS-NSF Regional Conference Series in Applied Mathematics. Philadelphia, PA:SIAM, 1992.

[5] J. S. Lee, Digital Image Enhancement and Noise Filtering by use of Local Statistics, IEEE Trans. on Pattern Anal. and Machine Intell., vol. 2, pp. 165, 1980.

[6] Martin Welk, Joachim Weickert, et al, "Median and Related Local Filters for Tensor-Valued Images", Tensor Signal Processing, 87:291-308, 2007.

[7] M. K. Mihcak, et al, "Low-complexity image denoising based on statistical modeling of wavelet coefficients," IEEE Signal Processing Letters, Vol. 6, Dec. 1999.

[8] P. Moulin and J. Liu, "Analysis of Multiresolution Image Denoising Schemes Using Generalized Gaussian and Complexity Priors," IEEE Trans. on Information Theory, Vol. 45, No. 3, pp. 909-919, April 1999.

[9] P. Besbeas, I. D. Fies and T. Sapatinas, A comparative simulation study of wavelet shrinkage estimators for Poisson counts, Int. Stats Rev., Vol. 72, pp. 209, 2004.

[10] R. Chan, C. Ho, and M. Nikolova, "Salt-and-pepper noise removal by median-type noise detectors and edge-preserving regularization," IEEE Trans. Image Processing, vol. 14, no. 10, pp. 1479-1485, 2005.

[11] R.C Gonzalez \& R.E Woods, DIPUM Toolbox.

[12] R.R. Coifman and D.L. Donoho, "Translation Invariant Denoising", Lecture Notes in Statistics, pp. 125-150, Springer Verlag, 1995.

[13] S. Mallat, "A theory for multiresolution signal decomposition: The wavelet representation", IEEE Trans. Pattern Anal. Machine Intell., vol. 11, 1989.

[14] __, "Analysis of Wavelet Family with Frobenius Norm for the Removal of Noise.", ICALIP '10, Accepted.

[15] T.F Chan and K. Chen, An optimization-based multilevel algorithm for total variation image denoising, SIAM J. Multiscale Modeling and Simulations, 5, pp. 615, 2006.

[16] T. Rockafellar, Convex Analysis, volume 224 of Grundlehren der mathematischen Wissenschaften. Princeton University Press, second edition, 1983.

[17] Y. Hawwar and A. Reza, "Spatially adaptive multiplicative noise image denoising technique," IEEE Trans. on Image Processing, vol. 11, pp. 1397, Dec. 2002. 